\title{
Supplementary information for \\ Identification of druggable kinase target conformations using Markov model metastable states analysis of apo-Abl
}

\author{
Fabian Paul ${ }^{1}$, Yilin Meng ${ }^{1}$, Benoît Roux ${ }^{1 *}$
}

1: Department of Biochemistry and Molecular Biology, University of Chicago, Chicago, IL, 60637-1454, USA

*: roux@uchicago.edu

\section{Suppl. Note 1 Molecular dynamics simulations}

The molecular dynamic simulations in ref. [7] were initiated from a multitude of structures (conformations), some of them directly taken form the PDB and some modeled on homologous kinases. The simulated kinase domain consists of residues W235-T498 (c-Abl splice 1a numbering). The Tyrosine in the A-loop was modeled as unphosphorylated. The Asp381 in the DFG-motif was modeled as deprotonated. The conformational ensemble was further enriched by "piecewise mixing of conformational variants" [7]. Eight $\mathrm{Na}^{+}$ions were add to neutralize the protein charge.

The conformational space was further explored by running $772 \mu$ s of MD simulations in very short trajectories (number of trajectories=7968, median length of single trajectory $100 \mathrm{~ns}$ ) using the Amberff-14SB [4] force field. A Markov state model was estimated at a lag time of $40 \mathrm{~ns}$ (400 steps) using MSMBuilder [1] version 3.

\section{Suppl. Note 2 Order parameters used to describe the conformations of the sub-domains}

We describe the conformations and conformational ensembles using a few selected distances (see suppl. table 1 and suppl. Fig. 1). These are the same distances as used in reference [7] for computing potentials of mean force.

\begin{tabular}{|c|c|c|}
\hline distance number & atoms & structural element \\
\hline \hline 1 & $\mathrm{C}_{\alpha}$-L248 and $\mathrm{C}_{\alpha}$-Y253 & P-loop \\
\hline 2 & $\mathrm{C}_{\alpha}$-G249 and $\mathrm{C}_{\alpha^{-}}$-Q252 & P-loop \\
\hline 3 & $\mathrm{C}_{\gamma}$-D381 $\mathrm{C}_{\alpha}$-N368 & DFG motif \\
\hline 4 & $\mathrm{C}_{\gamma}$-F382 $\mathrm{C}_{\alpha}$-I293 & DFG motif \\
\hline 5 & $\mathrm{C}_{\zeta}$-R386 $\mathrm{C}_{\delta^{-}}$-E286 & $\alpha$ C helix, salt bridge \\
\hline 6 & $\mathrm{~N}_{\zeta^{-}}$-K271 $\mathrm{C}_{\delta^{-}}$-E286 & $\alpha$ C helix, salt bridge \\
\hline 7 & O-Gly383 and N-Arg386 & A-loop (N-terminal helix) \\
\hline
\end{tabular}

Suppl. Table 1: Order parameters used for the characterization of Abl kinase sub-domain states in this work and in ref. [7].

For each metastable state, 1000 representative MD snapshots are saved by sampling with replacement form the metastable distributions [10] of each metastable state. To produce a smoothed contour for each state, a 2-D Gaussian kernel density estimate [11] was determined from the 1000 representative snapshots for pairs of observables. For visualization, the density iso-value was identified that surrounds $90 \%$ of the probability mass and simultaneously surrounds the smallest area.

N. B. This set of order parameters is distinct from the set of order parameters that has used for the definition of MSM microstates. For those order parameters, refer to table S1 in the supplementary information of ref. [7]. 


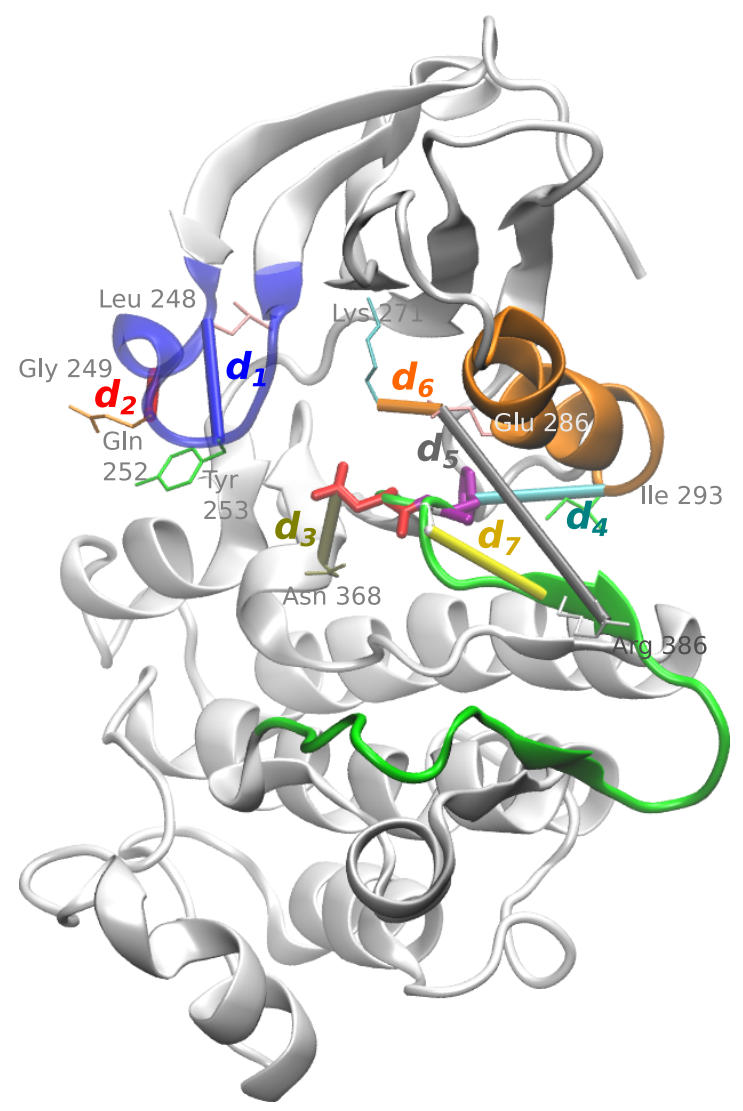

Suppl. Fig. 1: Distances used for the characterization of Abl kinase sub-domain states in this work and in ref. [7]. Residues that are involved in the distances are shown as sticks. Distances are visualized as cylinders that connect the endpoints.

\section{Suppl. Note 3 Metastable macrostate decomposition}

We reanalyze the Markov state model (MSM) that we published previously. [7] This MSM was built by clustering a collective variable space into 10000 microstates with the $k$-means algorithm. The collective variable space was defined by a few hand-selected order parameters for the P-loop, the $\alpha \mathrm{C}$ helix and the DFG motif as well as 5 time-independent components (tICs) that describe the dynamics of the A-loop (see supplementary documents of [7]). The lag time of that Markov model is $40 \mathrm{~ns}$.

We partition the state space into 30 metastable states using the PCCA+ algorithm as implemented in MSMBuilder. [1] The exact number of macrostates is to some degree arbitrary. The implied timescale plot in suppl. Fig. 8 shows that the timescales decay quickly with rank and that the single-microsecond regime can be reached by clustering in the space of the 30 dominant relaxtion modes. Still, there remains some uncertainty about the robustness of the results with respect to the particular choice of the number 30. We therefore repeated the PCCA+ clustering with 20 and 40 metastable states and analyzed the results in the same way as reported in the main text for the 30-macrostate decomposition. In the following we summarize the main differences between the decompositions. Generally we find that the exact number of states does not affect the conclusions drawn in the main text.

Mis-folded and non-classical DFG states persist at all resolutions: there are 5 mis-folded states and one non-classical DFG state when clustering with 20 states, 6 mis-folded states and 3 non-classical DFG states when clustering with 30 states and 7 mis-folded states and 4 non-classical DFG states when clustering with 40 states. (See. suppl. Tabs. 12 and 11.)

The three PCCA states 13, 14, 16 obtained when clustering with 20 states (see suppl. Tab. 9) persist no matter if we cluster with 20 states, 30 states (see PCCA states 2, 14, 25 in main text Tab. 2) or 40 states (see PCCA states 33, 34, 36 in suppl. Tab. 10).

X-ray structures that were at an RMSD of $2.5 \AA$ and above move into the $0 \AA$ to $2.5 \AA$ range as the number of PCCA 
macrostates is increased. For instance, crystal structures $2 \mathrm{PYY}$ and $1 \mathrm{SNX}$ are in the $2.5 \AA$ to $3.0 \AA$ range when clustering with 20 states (see suppl. Tab. 9). As the number of PCCA states is increased to 30, 2PYY moves to the $0 \AA$ to $2.5 \AA$ range (see main text Tab. 2). Eventually as the number of states is increased to 40, both $2 \mathrm{PYY}$ and $1 \mathrm{SNX}$ have moved to the $0 \AA$ to $2.5 \AA$ range of two different PCCA states (see states 7, 28 in suppl. Tab. 10). Such effects are expected since the PCCA states become more structurally defined, as their number is increased.

Some crystal structures like 6FNG, 2OG8, and 5NKA only appear close to metastable states when clustering with 30 PCCA states or more. As we already show in main text Figs. 10d, f, h the P-loop conformation in these crystal structures deviates from the respective PCCA states. Only by the equal weighting of all flexible structural elements in the RMSD computation, the X-ray structures can be considered "close" to the PCCA states. So that assignment can be considered borderline but this is already transparent in the main text (see main text Figs. 10d, f, h). The general effect that new close crystal structures appear is also expected, since PCCA clusters conformation by barrier height. Long-lived but possibly high-free energy states are made visible as the total number of states is increased.

With 40 states, some redundancy appears: X-ray structure 3PYY becomes the closest structure to states 2, 4, and 9. (See suppl. Tab. 10.) It appears that PCCA starts to resolve states more finely than reflected in the placement of available crystal structures throughout conformational space. Suppl. Fig. 7B shows that with 40 PCCA states, details of A-loop conformation and $\alpha \mathrm{C}$ orientation are more finely resolved than with 30 PCCA states (main text Fig. 8). This is in agreement with our previous observation that the A-loop has a high number of metastable conformations that together make up the flexibility of the A-loop.

\section{Suppl. Note 4 Computation of minRMSD between crystal structures and MD snapshots}

To compute the RMSD between two structures with the same amino acid sequence, both structures are first RMSDaligned using the MdTraj software [5] and using a sub-selection of 85 amino acids that constitute the binding pocket according to ref. [12] excluding DFG motif, A-loop, P-loop and $\alpha \mathrm{C}$ (size of sub-selection: 63 amino acids). After RMSD-alignment, an averaged RMSD is computed that equally weights root means square deviations of the the Nterminal stem of the A-loop, the P-loop, the DFG motif and the $\alpha \mathrm{C}$ helix (see suppl. table 3 for details). All structure alignments and RMSD computations (except for the DFG motif) use the backbone atom coordinates and the $\mathrm{C}_{\beta}$ atom position (if resolved in the PDB structure). For the DFG motif, more side chain atoms had to be included in the selection, to better distinguish DFG-in and DFG-out structures.

In the cases where the proteins have different amino acid sequences, we used the sequence alignments published in the KLIFS database [12] (from the version that we downloaded on 12/9/2018) to match the residue numbers of different PDB files. minRMSD alignment and RMSD computation were performed after that. Since the number of resolved residues varies between PDB files, reported RMSD values have to be interpreted with caution. Crystal structures, with less the 5 resolved residues in the P-loop, less than 5 resolved residues in the $\alpha \mathrm{C}$ helix, or less than 3 resolved residues in the DFG motif were excluded from analysis. 

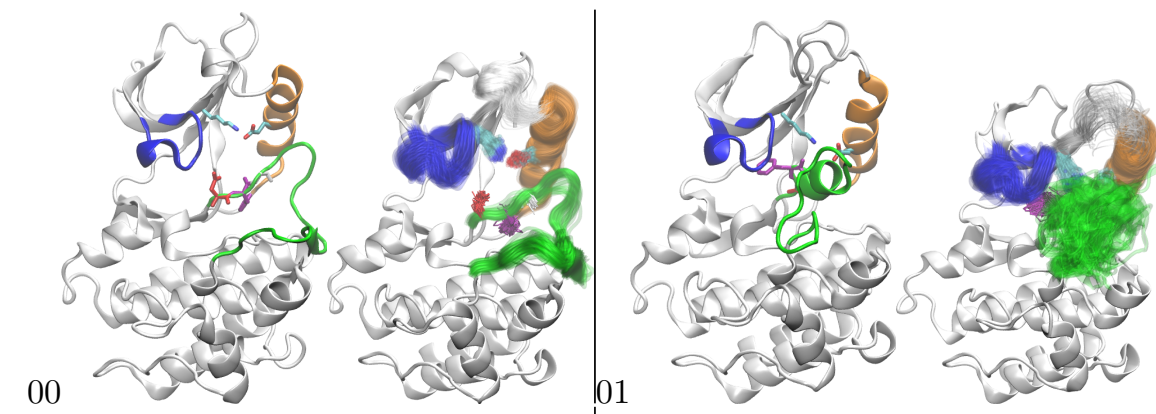

03

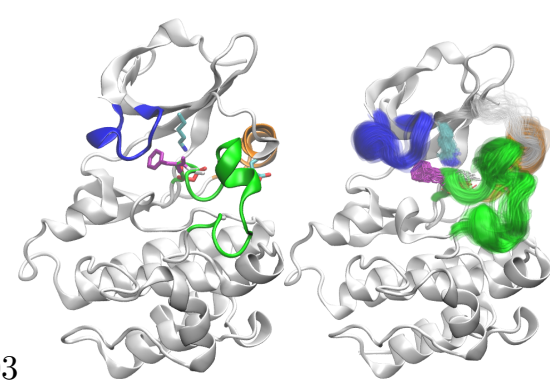

06

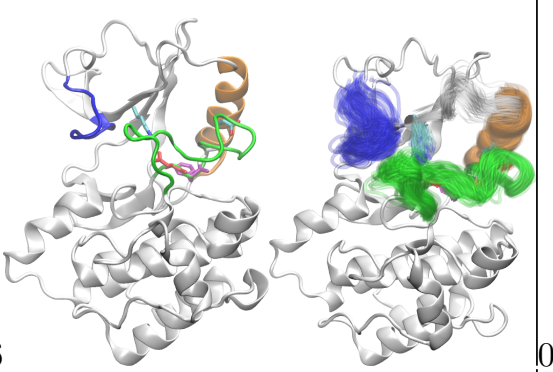

07

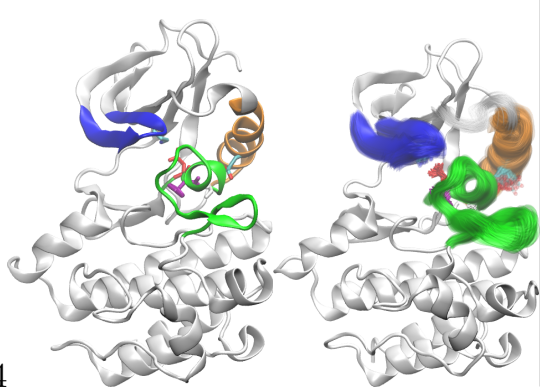

04

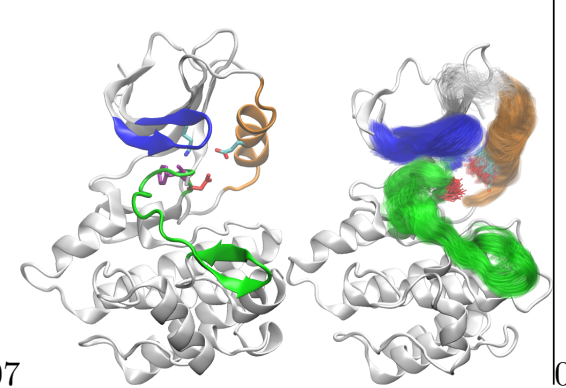

$\left.\right|_{05} ^{05}$
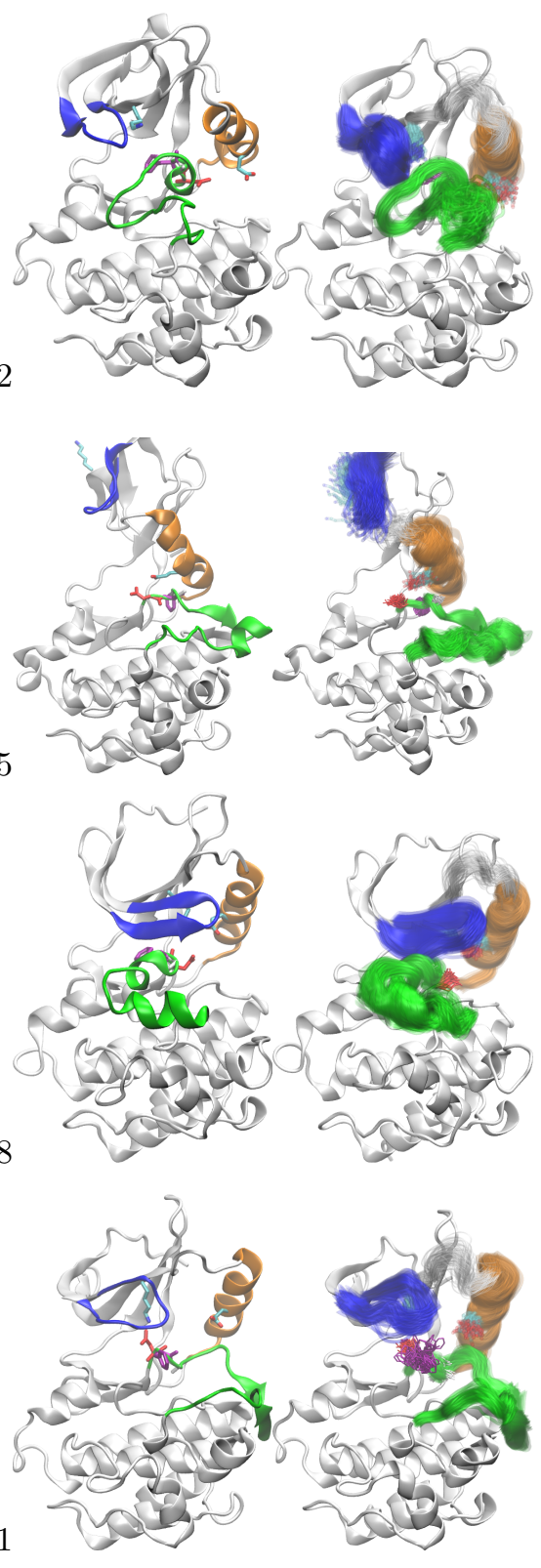

09
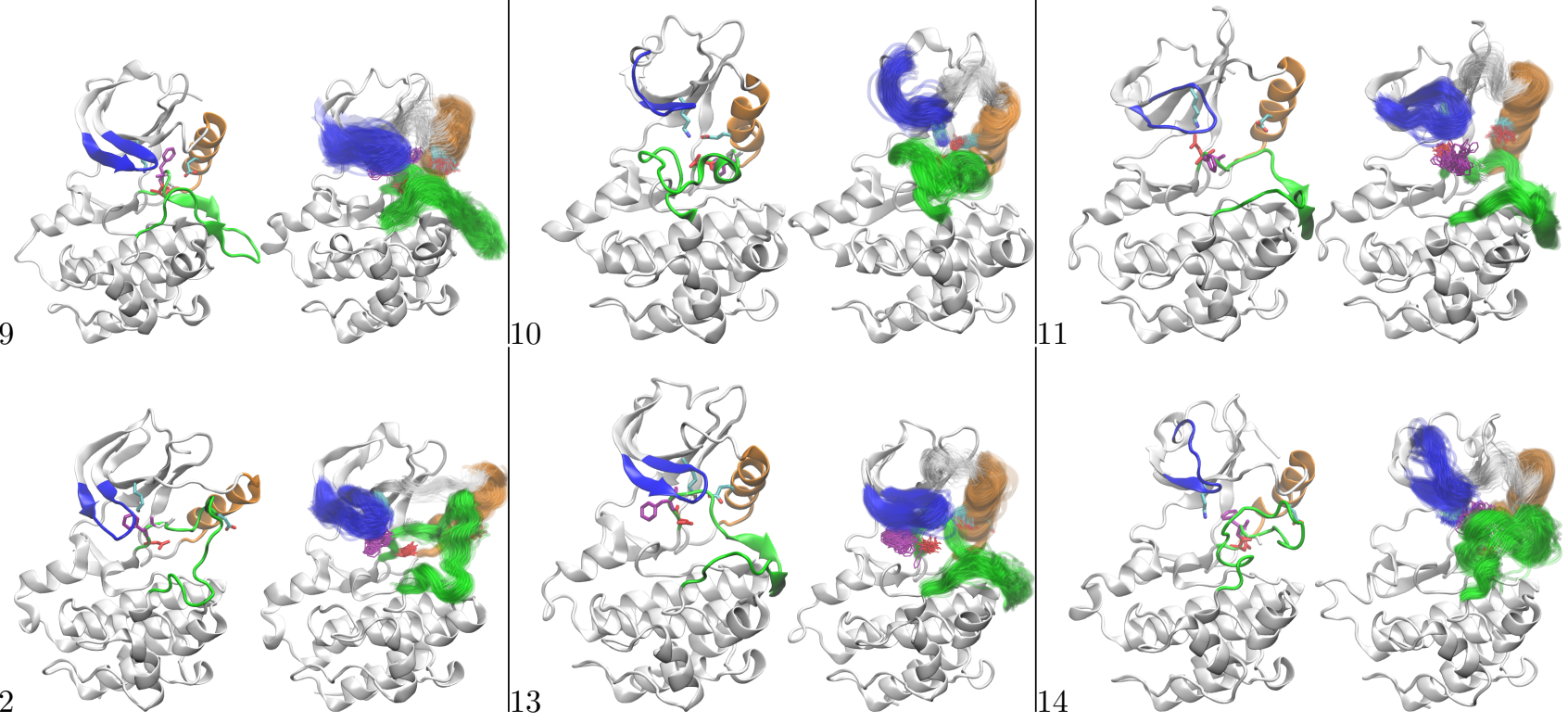

Suppl. Fig. 2: 30 PCCA macrostates from the MSM. Two images are shown for every macrostate, the macrostate medoid on the left and a superposition of 100 frames drawn from the ensemble on the right. Continued in next figure. 


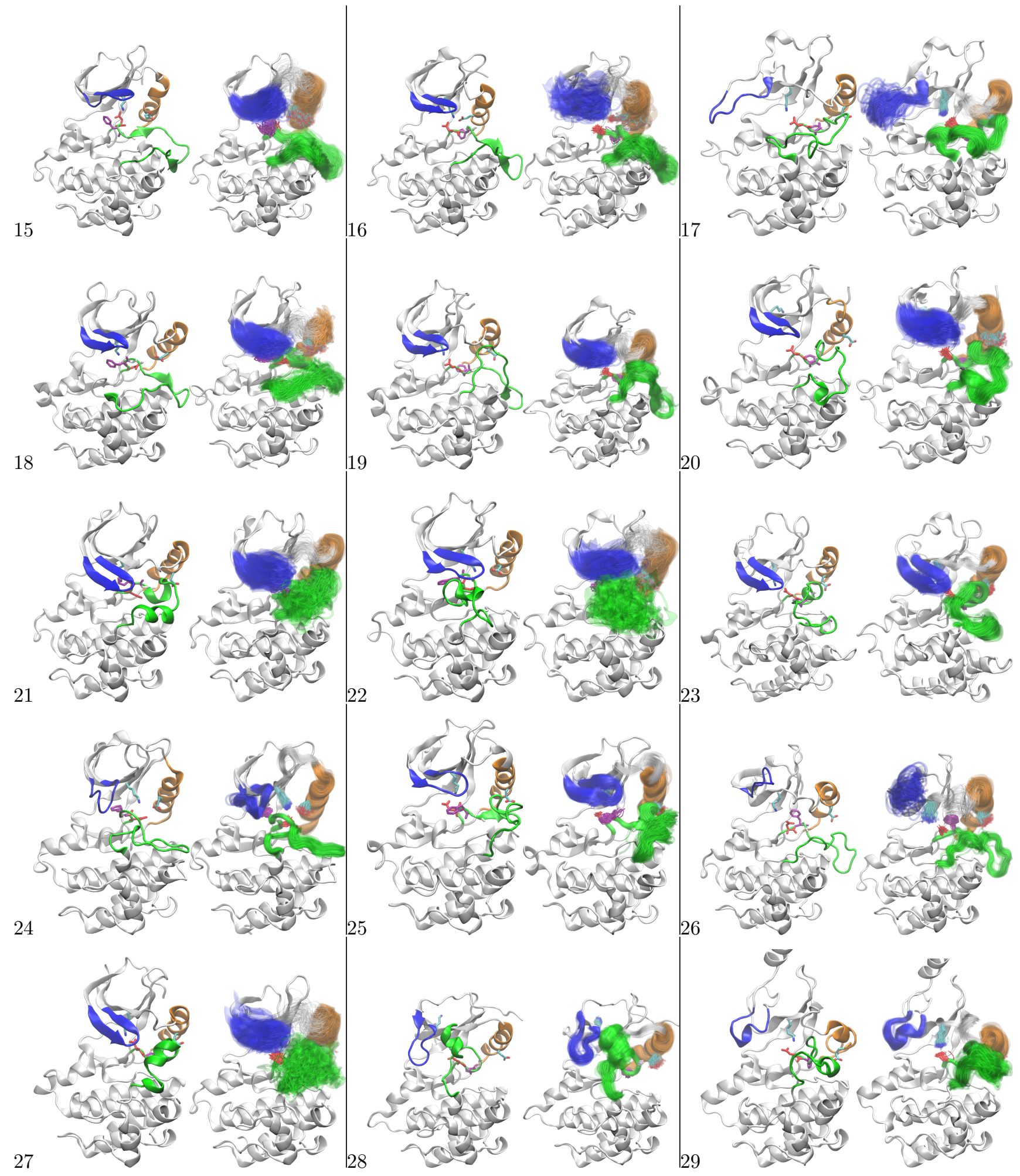

Suppl. Fig. 3: 30 PCCA macrostates from the MSM. Two images are shown for every macrostate, the macrostate medoid on the left and a superposition of 100 frames drawn from the ensemble on the right. Continuation from previous figure. 


\begin{tabular}{|c|c|c|c|}
\hline metastable state & DFG $_{\text {cluster }}{ }^{1}$ & DFG $_{\text {cluster }}^{2}$ & DFG subcluster $^{2}$ \\
\hline 0 & in & in ... & noise \\
\hline 1 & out & out inter & ................. noise \\
\hline 2 & out & out inter & B............... noise \\
\hline 3 & out & out & ............... nBA- noise \\
\hline 4 & out & in out ... & . \\
\hline 5 & - & in ... & 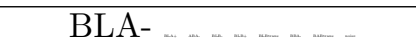 \\
\hline 6 & - & in ... & $\mathrm{BLA}+\ldots \ldots \ldots$ \\
\hline 7 & out & out & $\ldots$ BBA- - noise \\
\hline 8 & out & out & $\ldots$ BBA- $\ldots$ noise \\
\hline 9 & out & in out inter & BBA- BABtrans noise \\
\hline 10 & - & in $\ldots$ & BLA- $\ldots \ldots \ldots$ \\
\hline 11 & neither & in out intur & 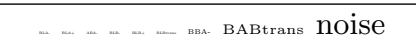 \\
\hline 12 & out & out & 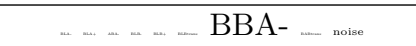 \\
\hline 13 & out & in out - & . \\
\hline 14 & - & out inter & BaBtrans noise \\
\hline 15 & out & in Out & $\ldots \ldots \ldots$ BABtrans noise \\
\hline 16 & in & in $\ldots$ & BLA- \\
\hline 17 & - & in ... & $\ldots$ BLB $+\ldots \ldots \ldots$ \\
\hline 18 & out & out & $\ldots$ BBA- $\ldots$ noise \\
\hline 19 & in & in ... & $\ldots$ \\
\hline 20 & in & in $\ldots$ & $\mathrm{BLA}+\ldots \mathrm{BLB}+\ldots \ldots$ \\
\hline 21 & out & Out ${ }_{\text {inter }}$ & B.............. BBA- noise \\
\hline 22 & out & in out & $\ldots$ BBA- - noise \\
\hline 23 & in & in $\ldots$ & BLB $+\ldots \ldots$ \\
\hline 24 & out & out inter & $\ldots$ BBA- - noise \\
\hline 25 & neither & out & noise \\
\hline 26 & neihter & inter & $\ldots \ldots \ldots \ldots$ noise \\
\hline 27 & in & in ... & $\ldots$ BLA+ $\ldots$ BLB $+\ldots \ldots$ noise \\
\hline 28 & in & in ... & $\mathrm{BLA}+\ldots \mathrm{BLB}+\ldots \ldots$ \\
\hline 29 & - & in ... & BLB- \\
\hline
\end{tabular}

Suppl. Table 2: Assignment of DFG conformational state to the manually defined clusters based on the distance pair $d_{3}, d_{4}$ (DFG cluster ${ }^{1}$ ) and assignment to the clusters and subclusters defined by Modi and Dunbrack [9] (DFG cluster ${ }^{2}$, DFG subcluster ${ }^{2}$ ). The manual analysis of the conformational states of the DFG motif may be compared with the more sophisticated classification introduced by Modi and Dunbrack. [9] The authors collected the conformations into three broad clusters: DFG-in, DFG-out and DFG-inter. The DFG-in class is further subdivided into the subclusters: BLA-, BLA+, ABA-, BLB-, BLB+ and BLBtrans. These sub-clusters are labeled with four-letter codes that encode the location of three consecutive backbone dihedral angles of $\mathrm{X}, \mathrm{D}$, and $\mathrm{F}$ amino acids in the Ramachandran plane, where $\mathrm{X}$ is the residue preceding the $\mathrm{DFG}$ motif $(\mathrm{A} \sim \alpha$-helical, $\mathrm{B} \sim \beta$-sheet, $\mathrm{L} \sim$ left-handed helical $)$ as well as the $\chi_{1}$ dihedral angle of the $\mathrm{F}$ side chain $\left(+\sim+60^{\circ},-\sim-60^{\circ}\right.$, trans $\left.\sim 180^{\circ}\right)$. Since each metastable state is an ensemble of conformations, the assignment between states and Modi-Dunbrack DFG clusters is fuzzy. We computed the probabilities $p_{i j}=\mathbb{P}($ cluster $i \mid$ state $j$ ) from 1000 representative frames sampled form each of the metastable states. Cluster labels are shown in this table with a font size that is proportional to $\sqrt{p_{i j}}$. BLA-corresponds to the catalytically active state and is typically observed in apo crystal structures, BLB+ approximately corresponds to the canonical "Src-like inactive" DFG-in conformation, BBA- corresponds to the DFG-out conformation, and BABtrans corresponds to the DFG-inter cluster. While the top-level clusters form a full partition of DFG conformational space, the sub-clusters are only defined for high-density core regions (i.e. regions with many similar crystal structures in the RSCB protein data bank). Conformations that fall outside the core regions were labeled as "noise". See main text for further details. 

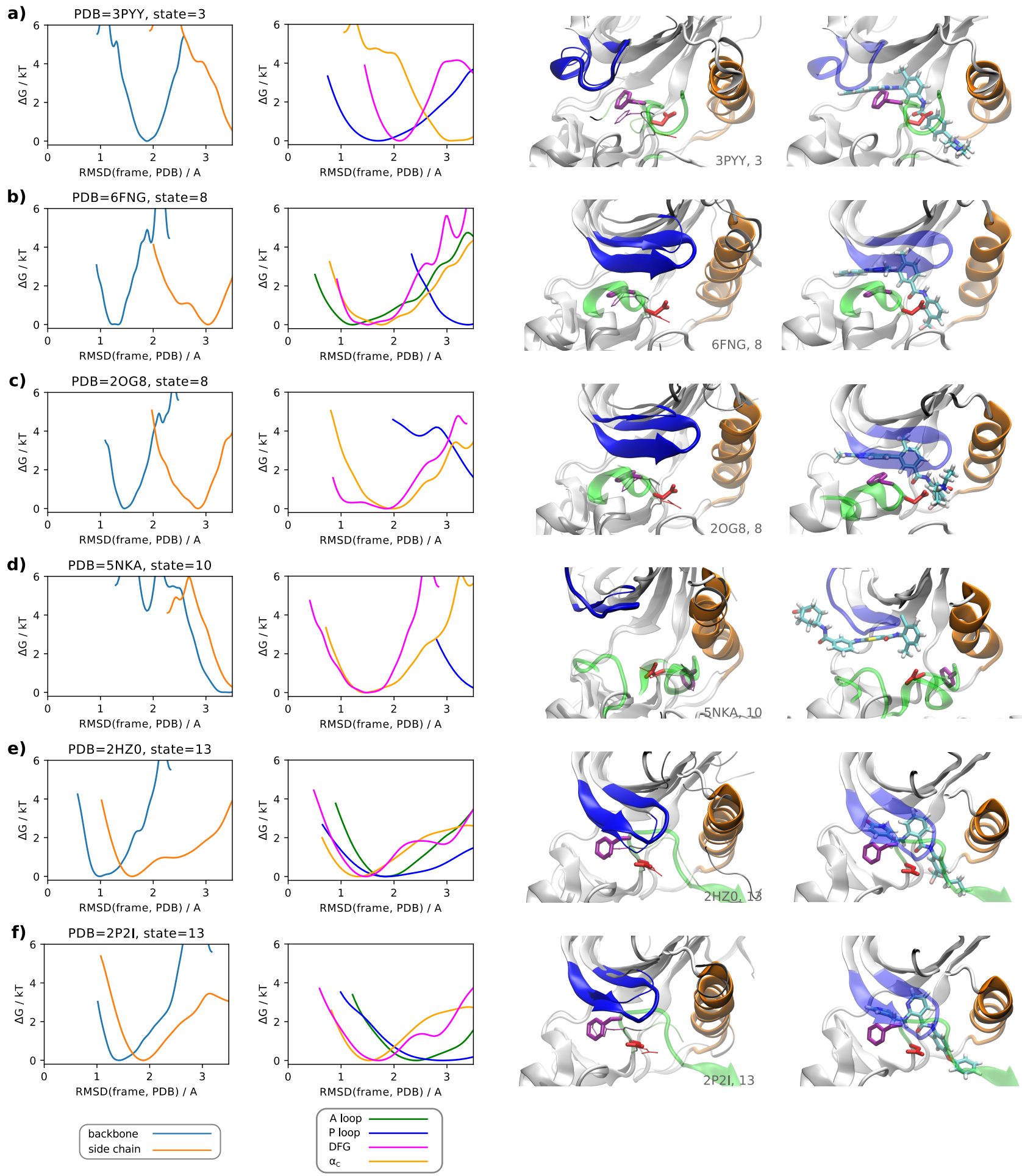

Suppl. Fig. 4: Same as main text Fig. 11 with added ligand information. The first column shows the RMSD of the residues that contact the ligand. RMSD was calculated separately for backbone and side chain atoms. The rightmost column shows the medoid structures of the metastable states superimposed to the coordinates of the ligand in the respective "close" crystal structure. Continued in suppl. Fig. 5. 

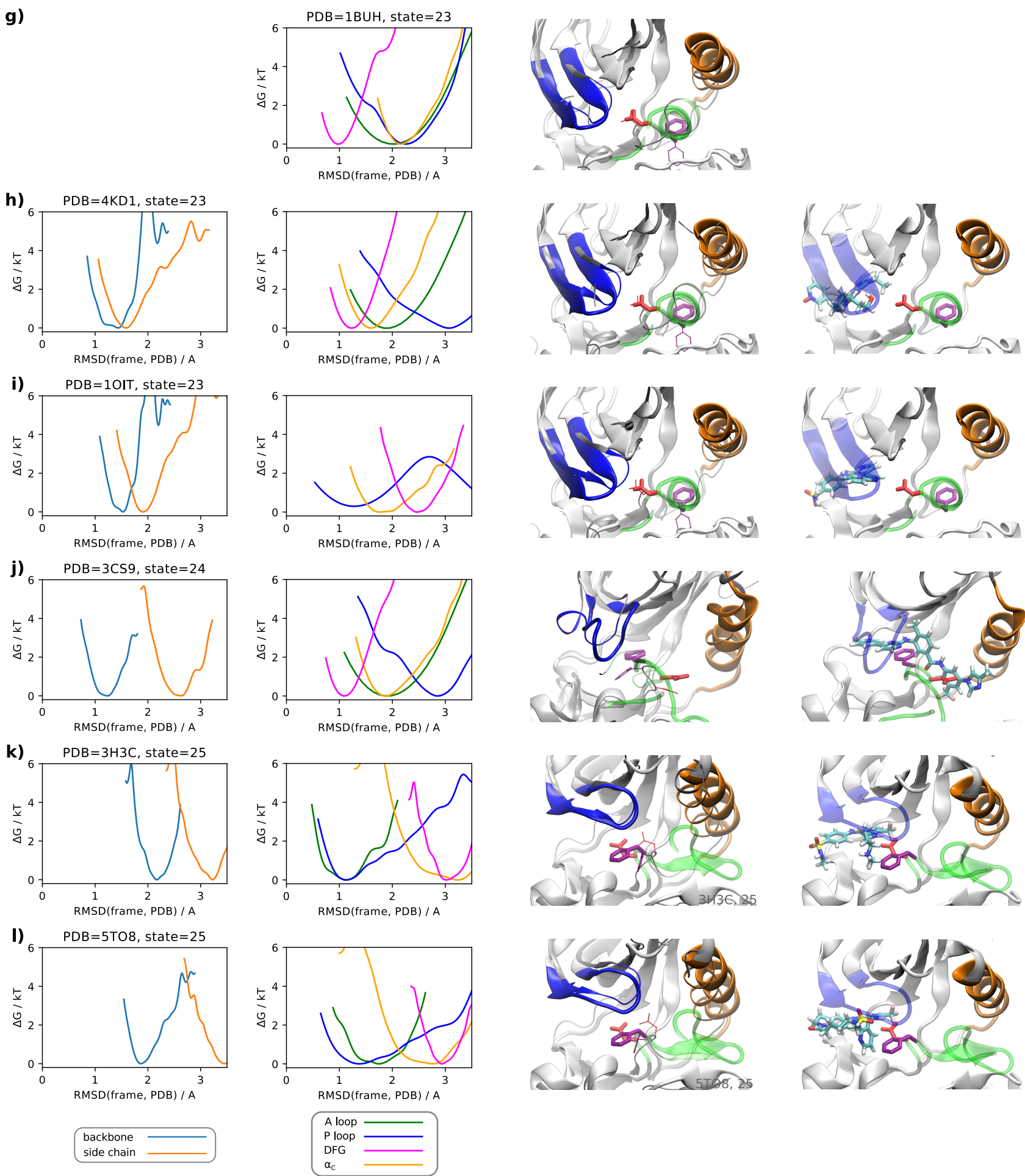

Suppl. Fig. 5: Continued from suppl. Fig. 4. 
metastable state

$\begin{array}{llllllllllllllllllllllllllllll}0 & 1 & 2 & 3 & 4 & 5 & 6 & 7 & 8 & 9 & 10 & 11 & 12 & 13 & 14 & 15 & 16 & 17 & 18 & 19 & 20 & 21 & 22 & 23 & 24 & 25 & 26 & 27 & 28 & 29\end{array}$

\begin{tabular}{|c|c|c|c|c|c|c|c|c|c|c|c|c|c|c|c|c|c|c|c|c|c|c|c|c|c|c|c|c|c|c|}
\hline 0 & 1.8 & 5.9 & 7.1 & 6.9 & 5.9 & 8.7 & 5.5 & 10.2 & 10.2 & 5.3 & 6.9 & 4.6 & 5.9 & 6.1 & 6.7 & 5.5 & 6.2 & 7.4 & 5.4 & 5.9 & 5.9 & 5.7 & 6.9 & 5.1 & 6.6 & 5.0 & 6.3 & 6.0 & 7.2 & 7.8 \\
\hline 1 & 5.9 & 3.6 & 4.4 & 3.6 & 5.3 & 10.4 & 6.7 & 7.7 & 7.4 & 6.6 & 5.8 & 6.5 & 5.9 & 6.1 & 6.4 & 7.3 & 8.3 & 7.4 & 6.3 & 8.4 & 6.3 & 5.4 & 5.7 & 5.6 & 4.1 & 6.5 & 8.7 & 6.7 & 6.4 & 6.7 \\
\hline 2 & 7.1 & 4.4 & 2.8 & 3.9 & 5.2 & 9.7 & 7.6 & 7.3 & 7.1 & 7.5 & 5.2 & 7.5 & 6.9 & 6.9 & 6.9 & 8.1 & 8.5 & 8.1 & 7.3 & 8.7 & 6.4 & 6.1 & 5.8 & 5.9 & 5.0 & 7.3 & 9.6 & 7.0 & 5.9 & 6.6 \\
\hline 3 & 6.9 & 3.6 & 3.9 & 2.1 & 5.5 & 10.4 & 7.2 & 7.6 & 7.3 & 7.7 & 6.1 & 7.5 & 6.5 & 6.4 & 7.0 & 8.2 & 8.9 & 7.3 & 7.2 & 9.0 & 6.7 & 6.1 & 6.0 & 6.2 & 4.0 & 7.1 & 9.4 & 7.2 & 5.9 & 6.2 \\
\hline & 5.9 & 5.3 & 5.2 & 5.5 & 1.3 & 9.1 & 6.2 & 7.4 & 7.1 & 5.4 & 4.4 & 4.9 & 5.4 & 5.3 & 5.3 & 5.4 & 6.6 & 7.1 & 5.2 & 6.2 & 4.3 & 4.3 & 4.7 & 4.2 & 5.6 & 4.7 & 7.7 & 5.0 & 6.0 & 6.8 \\
\hline 5 & 8.7 & 10.4 & 9.7 & 10.4 & 9.1 & 2.5 & 9.0 & 12.3 & 12.7 & 8.5 & 8.1 & 8.3 & 11.0 & 9.1 & 8.8 & 8.0 & 6.4 & 11.0 & 9.0 & 8.0 & 9.2 & 10.4 & 10.3 & 9.8 & 9.9 & 8.3 & 8.1 & 9.6 & 11.6 & 10.2 \\
\hline$\epsilon$ & 5.5 & 6.7 & 7.6 & 7.2 & 6.2 & 9.0 & 2.4 & 11.5 & 11.4 & 6.8 & 6.8 & 6.7 & 6.6 & 7.9 & 6.6 & 6.8 & 7.4 & 5.4 & 6.9 & 6.2 & 5.7 & 6.2 & 7.9 & 5.0 & 7.2 & 6.3 & 6.5 & 6.1 & 6.6 & 7.1 \\
\hline 7 & 10.2 & 7.7 & 7.3 & 7.6 & 7.4 & 12.3 & 11.5 & 2.2 & 3.0 & 9.1 & 7.4 & 8.2 & 9.8 & 6.6 & 8.6 & 9.3 & 10.3 & 12.7 & 8.4 & 11.6 & 9.0 & 8.6 & 6.3 & 9.4 & 6.8 & 8.8 & 12.3 & 9.0 & 9.6 & 11.1 \\
\hline 8 & 10.2 & 7.4 & 7.1 & 7.3 & 7.1 & 12.7 & 11.4 & 3.0 & 1.6 & 8.9 & 7.2 & 8.3 & 9.5 & 6.6 & 8.6 & 9.4 & 10.5 & 12.5 & 8.3 & 11.5 & 8.8 & 8.3 & 6.0 & 9.1 & 6.7 & 8.7 & 12.6 & 8.9 & 9.3 & 10.8 \\
\hline 9 & 5.3 & 6.6 & 7.5 & 7.7 & 5.4 & 8.5 & 6.8 & 9.1 & 8.9 & 2.8 & 7.2 & 4.4 & 5.4 & 5.0 & 6.0 & 4.5 & 5.1 & 9.1 & 3.3 & 5.2 & 6.2 & 5.0 & 6.3 & 5.4 & 7.0 & 4.2 & 6.0 & 6.1 & 8.9 & 9.6 \\
\hline 10 & 6.9 & 5.8 & 5.2 & 6.1 & 4.4 & 8.1 & 6.8 & 7.4 & 7.2 & 7.2 & 2.0 & 6.8 & 7.4 & 6.5 & 5.7 & 7.0 & 6.7 & 8.0 & 7.1 & 7.9 & 5.4 & 6.1 & 5.6 & 5.8 & 5.9 & 6.8 & 8.7 & 5.9 & 6.8 & 7.0 \\
\hline 11 & 4.6 & 6.5 & 7.5 & 7.5 & 4.9 & 8.3 & 6.7 & 8.2 & 8.3 & 4.4 & 6.8 & 1.8 & 6.3 & 4.7 & 5.9 & 4.4 & 5.8 & 9.0 & 4.4 & 6.1 & 6.2 & 5.7 & 6.0 & 5.7 & 6.6 & 3.3 & 6.5 & 5.8 & 8.4 & 9.0 \\
\hline 12 & 5.9 & 5.9 & 6.9 & 6.5 & 5.4 & 11.0 & 6.6 & 9.8 & 9.5 & 5.4 & 7.4 & 6.3 & 2.3 & 6.1 & 6.4 & 6.4 & 8.0 & 8.3 & 5.2 & 7.4 & 6.6 & 4.0 & 6.5 & 5.2 & 7.1 & 6.1 & 7.9 & 6.1 & 7.2 & 8.8 \\
\hline 13 & 6.1 & 6.1 & 6.9 & 6.4 & 5.3 & 9.1 & 7.9 & 6.6 & 6.6 & 5.0 & 6.5 & 4.7 & 6.1 & 2.2 & 6.4 & 5.8 & 6.4 & 10.1 & 4.4 & 7.5 & 6.6 & 5.7 & 5.5 & 6.4 & 5.4 & 5.3 & 8.6 & 6.6 & 9.1 & 9.7 \\
\hline 1 & 6.7 & 6.4 & 6.9 & 7.0 & 5.3 & 8.8 & 6.6 & 8.6 & 8.6 & 6.0 & 5.7 & 5.9 & 6.4 & 6.4 & 5.1 & 6.1 & 7.3 & 8.5 & 6.0 & 7.5 & 6.1 & 5.6 & 6.2 & 6.2 & 6.6 & 5.9 & 7.5 & 6.3 & 8.3 & 8.5 \\
\hline 15 & 5.5 & 7.3 & 8.1 & 8.2 & 5.4 & 8.0 & 6.8 & 9.3 & 9.4 & 4.5 & 7.0 & 4.4 & 6.4 & 5.8 & 6.1 & 4.3 & 5.6 & 9.1 & 4.8 & 5.3 & 6.5 & 5.9 & 6.7 & 6.1 & 7.5 & 4.3 & 5.9 & 6.3 & 9.2 & 9.5 \\
\hline 16 & 6.2 & 8.3 & 8.5 & 8.9 & 6.6 & 6.4 & 7.4 & 10.3 & 10.5 & 5.1 & 6.7 & 5.8 & 8.0 & 6.4 & 7.3 & 5.6 & 4.7 & 9.5 & 5.7 & 5.7 & 7.1 & 7.2 & 7.8 & 6.9 & 8.2 & 5.7 & 6.6 & 7.0 & 9.8 & 9.7 \\
\hline 17 & 7.4 & 7.4 & 8.1 & 7.3 & 7.1 & 11.0 & 5.4 & 12.7 & 12.5 & 9.1 & 8.0 & 9.0 & 8.3 & 10.1 & 8.5 & 9.1 & 9.5 & 3.1 & 9.1 & 8.1 & 6.7 & 7.5 & 9.2 & 5.9 & 8.2 & 8.4 & 8.4 & 7.5 & 6.6 & 5.4 \\
\hline 18 & 5.4 & 6.3 & 7.3 & 7.2 & 5.2 & 9.0 & 6.9 & 8.4 & 8.3 & 3.3 & 7.1 & 4.4 & 5.2 & 4.4 & 6.0 & 4.8 & 5.7 & 9.1 & 3.6 & 5.8 & 6.2 & 4.9 & 6.0 & 5.4 & 6.6 & 4.2 & 6.9 & 6.1 & 8.8 & 9.5 \\
\hline 19 & 5.9 & 8.4 & 8.7 & 9.0 & 6.2 & 8.0 & 6.2 & 11.6 & 11.5 & 5.2 & 7.9 & 6.1 & 7.4 & 7.5 & 7.5 & 5.3 & 5.7 & 8.1 & 5.8 & 1.8 & 6.2 & 6.9 & 8.2 & 5.2 & 8.4 & 5.2 & 5.6 & 6.6 & 8.9 & 9.7 \\
\hline 20 & 5.9 & 6.3 & 6.4 & 6.7 & 4.3 & 9.2 & 5.7 & 9.0 & 8.8 & 6.2 & 5.4 & 6.2 & 6.6 & 6.6 & 6.1 & 6.5 & 7.1 & 6.7 & 6.2 & 6.2 & 3.5 & 5.6 & 6.0 & 4.3 & 6.5 & 6.0 & 7.9 & 5.5 & 6.3 & 6.8 \\
\hline 21 & 5.7 & 5.4 & 6.1 & 6.1 & 4.3 & 10.4 & 6.2 & 8.6 & 8.3 & 5.0 & 6.1 & 5.7 & 4.0 & 5.7 & 5.6 & 5.9 & 7.2 & 7.5 & 4.9 & 6.9 & 5.6 & 3.2 & 5.5 & 4.1 & 6.8 & 5.4 & 7.6 & 5.2 & 6.8 & 8.3 \\
\hline 22 & 6.9 & 5.7 & 5.8 & 6.0 & 4.7 & 10.3 & 7.9 & 6.3 & 6.0 & 6.3 & 5.6 & 6.0 & 6.5 & 5.5 & 6.2 & 6.7 & 7.8 & 9.2 & 6.0 & 8.2 & 6.0 & 5.5 & 5.1 & 6.1 & 6.0 & 6.2 & 9.3 & 6.3 & 7.6 & 8.4 \\
\hline 23 & 5.1 & 5.6 & 5.9 & 6.2 & 4.2 & 9.8 & 5.0 & 9.4 & 9.1 & 5.4 & 5.8 & 5.7 & 5.2 & 6.4 & 6.2 & 6.1 & 6.9 & 5.9 & 5.4 & 5.2 & 4.3 & 4.1 & 6.1 & 1.1 & 6.7 & 5.0 & 7.0 & 4.5 & 5.3 & 7.2 \\
\hline & 6.6 & 4.1 & 5.0 & 4.0 & 5.6 & 9.9 & 7.2 & 6.8 & 6.7 & 7.0 & 5.9 & 6.6 & 7.1 & 5.4 & 6.6 & 7.5 & 8.2 & 8.2 & 6.6 & 8.4 & 6.5 & 6.8 & 6.0 & 6.7 & 1.6 & 6.7 & 9.0 & 7.5 & 7.4 & 7.0 \\
\hline 25 & 5.0 & 6.5 & 7.3 & 7.1 & 4.7 & 8.3 & 6.3 & 8.8 & 8.7 & 4.2 & 6.8 & 3.3 & 6.1 & 5.3 & 5.9 & 4.3 & 5.7 & 8.4 & 4.2 & 5.2 & 6.0 & 5.4 & 6.2 & 5.0 & 6.7 & 1.5 & 6.3 & 5.7 & 8.4 & 8.7 \\
\hline & 6.3 & 8.7 & 9.6 & 9.4 & 7.7 & 8.1 & 6.5 & 12.3 & 12.6 & 6.0 & 8.7 & 6.5 & 7.9 & 8.6 & 7.5 & 5.9 & 6.6 & 8.4 & 6.9 & 5.6 & 7.9 & 7.6 & 9.3 & 7.0 & 9.0 & 6.3 & 2.9 & 7.5 & 9.2 & 9.7 \\
\hline 27 & 6.0 & 6.7 & 7.0 & 7.2 & 5.0 & 9.6 & 6.1 & 9.0 & 8.9 & 6.1 & 5.9 & 5.8 & 6.1 & 6.6 & 6.3 & 6.3 & 7.0 & 7.5 & 6.1 & 6.6 & 5.5 & 5.2 & 6.3 & 4.5 & 7.5 & 5.7 & 7.5 & 5.6 & 6.7 & 8.2 \\
\hline & 7.2 & 6.4 & 5.9 & 5.9 & 6.0 & 11.6 & 6.6 & 9.6 & 9.3 & 8.9 & 6.8 & 8.4 & 7.2 & 9.1 & 8.3 & 9.2 & 9.8 & 6.6 & 8.8 & 8.9 & 6.3 & 6.8 & 7.6 & 5.3 & 7.4 & 8.4 & 9.2 & 6.7 & 1.9 & 6.3 \\
\hline & 7.8 & 6.7 & 6.6 & 6.2 & 6.8 & 10.2 & 7.1 & 11.1 & 10.8 & 9.6 & 7.0 & 9.0 & 8.8 & 9.7 & 8.5 & 9.5 & 9.7 & 5.4 & 9.5 & 9.7 & 6.8 & 8.3 & 8.4 & 7.2 & 7.0 & 8.7 & 9.7 & 8.2 & 6.3 & 1.9 \\
\hline
\end{tabular}

Suppl. Fig. 6: Average RMSD between random pairs of frames selected form different metastable states (off-diagonal elements) and average RMSD between random frames selected form the same metastable state (diagonal elements). Floating point values are the average RMSD reported in units of Angstroms. The RMSD was computed in the same manner as the RMSD between X-ray structures and metastable states. Instead of aligning all frames to the rigid subdomains in the crystal structures, pair of snapshots were aligned to each other (using the same set of atoms for alignment). 
A)

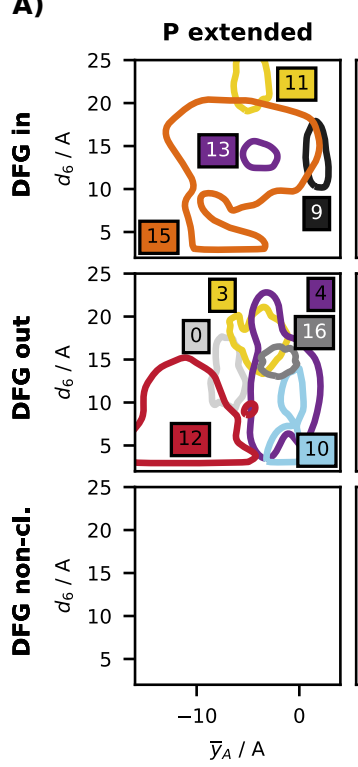

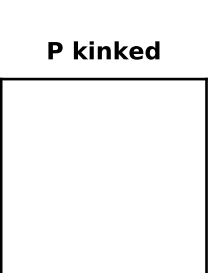
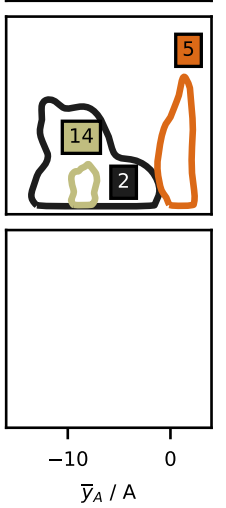

P disordered/

coil
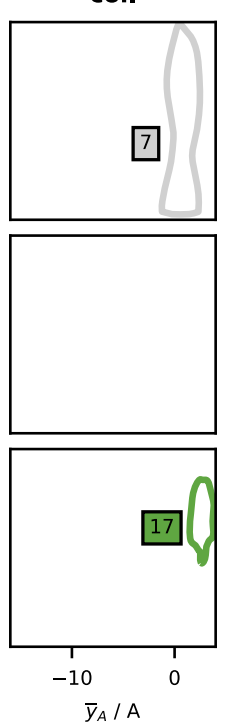

B)
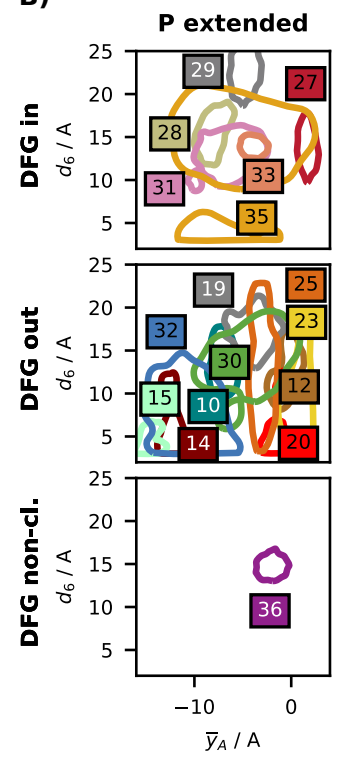
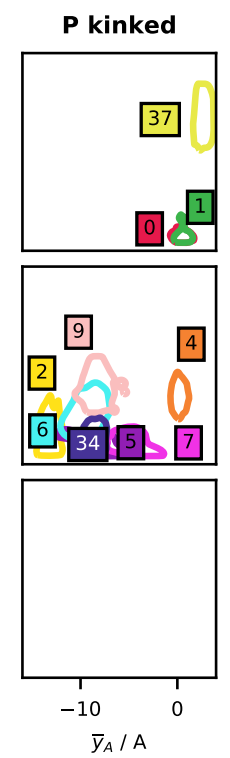

P disordered/

coil
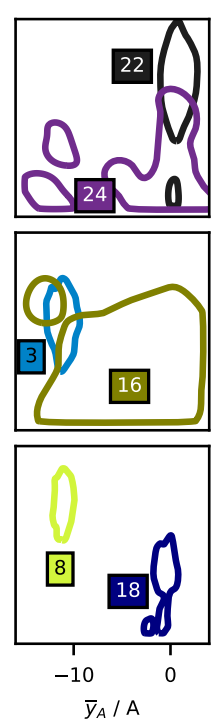

Suppl. Fig. 7: Same as main text Fig. 8 but for a PCCA+ decompostion of the MSM into (A) 20 states and (B) 40 states. States were numbered independently in both analysis. Since PCCA+ is no hierarchical clustering method, there is in general no one-to-many relation between the states of two different decompositions, though the decomposion can in principle reflect the nature of hierarachical energy landscapes. [2]

a)

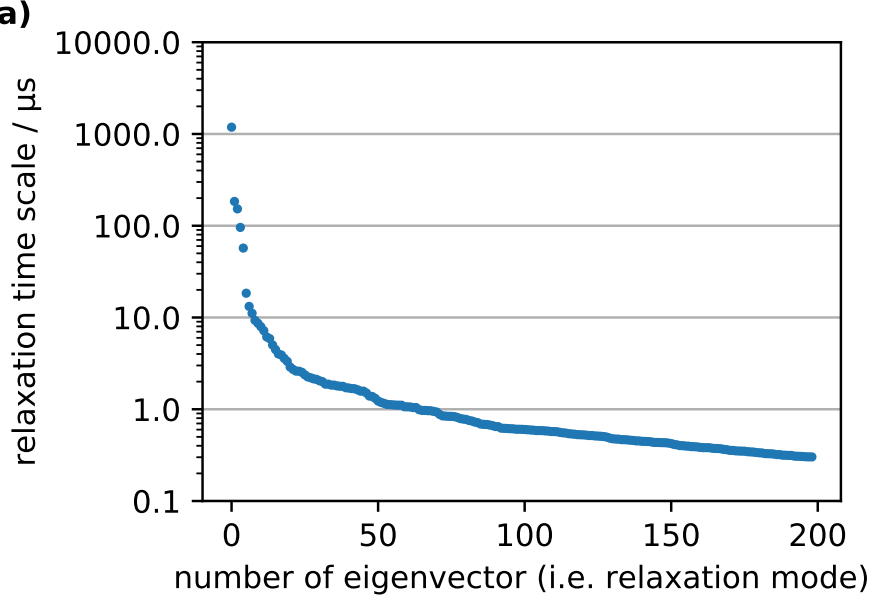

b)

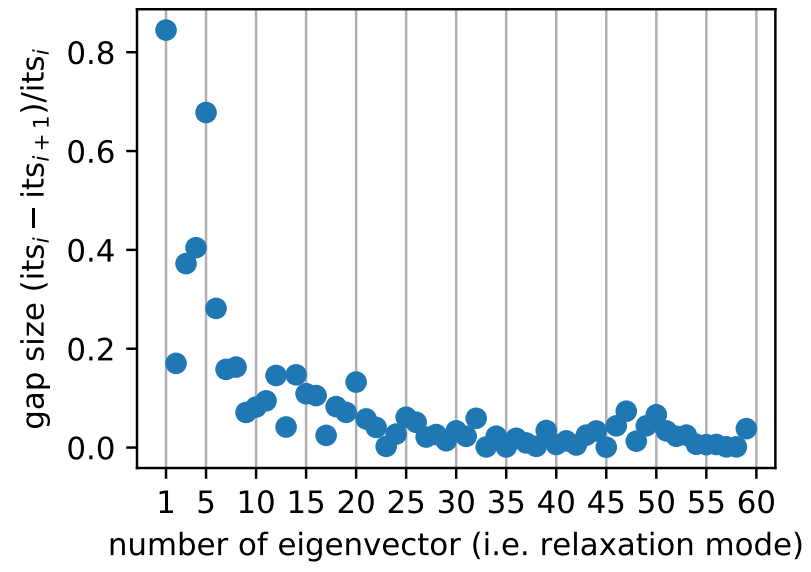

Suppl. Fig. 8: a) Dominant 200 implied timescales (relaxation timescales) of the full 10000-microstate MSM of Ref. [7] that was estimated at a lag time of $40 \mathrm{~ns}, \mathrm{~b}$ ) Relative differences of successive time scales indicate that there is a spectral gap after the first time scale and possibly a small spectral gap after the fifth time scale. 


\begin{tabular}{|c|c|c|c|}
\hline structural element & residues & atom types & weight \\
\hline \hline DFG motif & $381-383$ & $\mathrm{C}_{1} \mathrm{C}_{\alpha}, \mathrm{C}_{\beta}, \mathrm{C}_{\gamma}$ & $1 / 4$ \\
\hline P-loop & $248-257$ & $\mathrm{~N}, \mathrm{C}_{\alpha}, \mathrm{C}_{\beta}, \mathrm{C}, \mathrm{O}$ & $1 / 4$ \\
\hline$\alpha \mathrm{C}$ helix & $280-294$ & $\mathrm{~N}, \mathrm{C}_{\alpha}, \mathrm{C}_{\beta}, \mathrm{C}, \mathrm{O}$ & $1 / 4$ \\
\hline N-terminal stem of A-loop & $384-388$ & $\mathrm{~N}, \mathrm{C}_{\alpha}, \mathrm{C}_{\beta}, \mathrm{C}, \mathrm{O}$ & $1 / 4$ \\
\hline
\end{tabular}

Suppl. Table 3: Atom selection for RMSD computations

\begin{tabular}{|c|c|c|c|c|c|}
\hline & \multirow{2}{*}{\multicolumn{3}{|c|}{ Crystal structures at $x$ RMSD around the state }} \\
\hline & & & & & \\
\hline$s$ & DFG & P-loop & $0 \AA$ to $2.5 \AA$ & $2.5 \AA$ to $3.0 \AA$ & $3.0 \AA$ to $3.5 \AA$ \\
\hline 0 & in & kinked & & MET, HGK, FGFR4 & HGK, p38a, TRKA, FGFR1, INSR, + \\
\hline 1 & out & kinked & & ABL1 & LOK, p38a, AurB, ABL1, TRKA \\
\hline 2 & out & loop & & AurB & LOK, ABL1, p38a, ABL2 \\
\hline 3 & out & kinked & ABL1 & ABL1, AurB & p38a, ABL2, EphA3, ABL1, LOK \\
\hline 4 & out & ext. & & CaMK1a, EGFR, PYK2, CDK2, AurA, + & ErbB2, CSK, PKN1, EGFR, PAK1, + \\
\hline 5 & - & - & & & \\
\hline 6 & - & - & & & \\
\hline 7 & out & ext. & & PYK2, p38a, TRKA, KDR, LCK & EphA2, p38a, DDR1, EphB4, TRKB, + \\
\hline 8 & out & ext. & EphA2, LCK & PYK2, EphB4, CDK8, KDR, p38a, + & CDK8, PAK1, DDR1, p38a, TRKB, + \\
\hline 9 & out & ext. & & MAP3K5, EGFR & EGFR, BMX, CK1e, PKR, MAP3K5, + \\
\hline 10 & - & - & EphA2 & ITK, EphA7, EphA2, EphA3, FAK, + & MET, EGFR, EphB4, FAK, p110g, + \\
\hline 11 & neither & & & & PYK2, INSR \\
\hline 12 & out & ext./loop & & & ULK3, CaMK1a \\
\hline 13 & out & ext. & ABL1, KDR & KDR, RIPK2, TRKA, TAK1, PEK, + & KDR, TRKA, LOK, p38a, BRAF, + \\
\hline 14 & - & - & & & \\
\hline 15 & out & ext. & & & PYK2, RSK2, NEK2 \\
\hline 16 & in & disordered & & SRC, JAK2 & FYN, LCK, ULK1, CLK4, SRC, + \\
\hline 17 & - & - & & & \\
\hline 18 & out & ext. & & & EGFR, LOK, CK1e \\
\hline 19 & in & ext. & & MST4, CDK2, ITK & $\begin{array}{l}\text { FGFR4, ITK, TNIK, EGFR, TBK1, + } \\
\end{array}$ \\
\hline 20 & in & ext. & & EGFR, ITK, TBK1, CDK2 & EGFR, CDK2, p38a, PAK1, CDK6, + \\
\hline 21 & out & ext. & & & CSK \\
\hline 22 & out & ext. & & & p38a, TRKA \\
\hline 23 & in & ext. & BRK, CDC2, EGFR, ErbB4, BTK + & ACK, BTK, SRC, BRK, ErbB4, + & TNIK, EGFR, ITK, Wnk3, ErbB3, + \\
\hline 24 & out & kinked & ABL1 & ABL1, p38a & LOK, TRKA, ABL2, EphA3, RIPK2, + \\
\hline 25 & neither & ext. & PYK2 & PYK2, INSR, CDK2, RSK2 & CDK2, MST4, PYK2, RSK2, AurA + \\
\hline 26 & neither & kinked/loop & & & \\
\hline 27 & in & ext./loop & & ITK, EGFR, CDK2, TBK1, BRAF & CaMK1a, ZAP70, BRAF, PEK, CDK2 + \\
\hline 28 & in & loop & & MET & CDKL2, HGK, EGFR \\
\hline 29 & - & - & & & \\
\hline
\end{tabular}

Suppl. Table 4: Same as main text table 2 put showing the kinase type instead of the PDB identifier. 


\begin{tabular}{|c|c|c|c|c|c|}
\hline & & & Crystal & tructures at $x$ RMSD* arc & nd the state \\
\hline$s$ & DFG & P-loop & $0 \AA$ to $2.5 \AA$ & $2.5 \AA$ to $3.0 \AA$ & $3.0 \AA$ to $3.5 \AA$ \\
\hline 0 & in & kinked & & 03X, 3C8, 4QG, 5DF, -, + & $-,-, 4 \mathrm{UT}, 469, \mathrm{~V} 4 \mathrm{Z},+$ \\
\hline 1 & out & kinked & & NIL, STI & B96, DG7, 0LI, OLI, VX6, + \\
\hline 2 & out & coil & & VX6 & B96, 0LI, NIL, 0LI, 406, + \\
\hline 3 & out & kinked & STI & 406, NIL, OLI, OLI, VX6 & DG7, STI, 406, IFC, 66K, + \\
\hline 4 & out & ext. & & ATP, 9LL, LZD, -, AGS, + & $\mathrm{SCZ},-, 325,03 \mathrm{P}, 3 \mathrm{ZC},+$ \\
\hline 5 & - & - & & & \\
\hline 6 & - & - & & & \\
\hline 7 & out & ext. & & B96, Z83, GO7, RAJ, 1N8 & STI, DWT, FKY, AQZ, AQZ, + \\
\hline 8 & out & ext. & DWT, $1 \mathrm{~N} 8$ & B96, DWT, 0SR, 0SU, RAJ, + & 0SQ, FKY, 4OR, DI1, AQZ, + \\
\hline 9 & out & ext. & & STU, O44 & $633,630,8 \mathrm{BM}, 8 \mathrm{AM}, 63 \mathrm{~A},+$ \\
\hline 10 & - & - & $91 \mathrm{H}$ & 91E, PQC, FAV $,-, 8 \mathrm{ZW},+$ & QYT, DXK, JZW, -, QAQ, + \\
\hline 11 & neither & ext./coil & & & P1E, AGS, 7FM,-- \\
\hline 12 & out & ext./coil & & & $\mathrm{C} 87, \mathrm{ATP}$ \\
\hline 13 & out & ext. & GIN, 608 & $\mathrm{Z} 83,276, \mathrm{SR} 8,6 \mathrm{~K} 0, \mathrm{NG} 2,+$ & FKY, RAJ, GO7, G6I, 0T2, + \\
\hline 14 & - & - & & & \\
\hline 15 & out & ext. & & & 7FM, P1E, 349, 3JZ, 584, + \\
\hline 16 & in & disordered & & HET, BJG & STU, CG9, 3RF, X9G, 8X7, + \\
\hline 17 & - & - & & & \\
\hline 18 & out & ext. & & & $-,-, 8 \mathrm{BM}, \mathrm{O} 44,633,+$ \\
\hline 19 & in & ext. & & 0F2, GVD, 2VT, STU, 0G1, + & 6LF, 6LF, -, PQC, G6K, + \\
\hline 20 & in & ext. & & 9LL, ANP, LZD, -, 1FV, + & STU, L7A, STU, ITI, EZV, + \\
\hline 21 & out & ext. & & & STU \\
\hline 22 & out & ext. & & & Z83, GO7 \\
\hline 23 & in & ext. &,- HDT, 1QK, 1E8, 16K, + & OOS, -, 4C9, W32, ANP, + & $58 \mathrm{C}, \mathrm{X} 40,4 \mathrm{AU}, \mathrm{ACP}, 3 \mathrm{BM},+$ \\
\hline 24 & out & kinked & NIL & STI, NIL, 0LI, 406, 0LI, + & $\mathrm{B} 96,6 \mathrm{~K} 0, \mathrm{GXA}, 6 \mathrm{~K} 1,6 \mathrm{~K} 2,+$ \\
\hline 25 & neither & ext. & $\mathrm{P} 1 \mathrm{E}, 7 \mathrm{FM}$ & 3JZ, 349, -, LZ7, AGS, + & LZA, X4Z, -, YAM, WCX, + \\
\hline 26 & neither & kinked/coil & & & \\
\hline 27 & in & ext./coil & & -, STU, STU, ANP, ITI, + & ATP, 3I6, BX7, 324, ANP, + \\
\hline 28 & in & coil & & $03 \mathrm{X}$ & $4 \mathrm{QG},-, 3 \mathrm{C} 8,-, \mathrm{DKI},+$ \\
\hline 29 & - & - & & & \\
\hline
\end{tabular}

Suppl. Table 5: Same as main text table 2 put showing the ligand identifier instead of the PDB identifier. $\mathrm{STI}=$ imatinib, 406=bafetinib, $1 \mathrm{E} 8=$ ibrutinib, NIL=nilotinib, $0 \mathrm{LI}=$ ponatinib, $\mathrm{STU}=$ staurosporine, $\mathrm{B} 49=$ sunitinib, VX6=tozasertib, FMM=lapatinib.

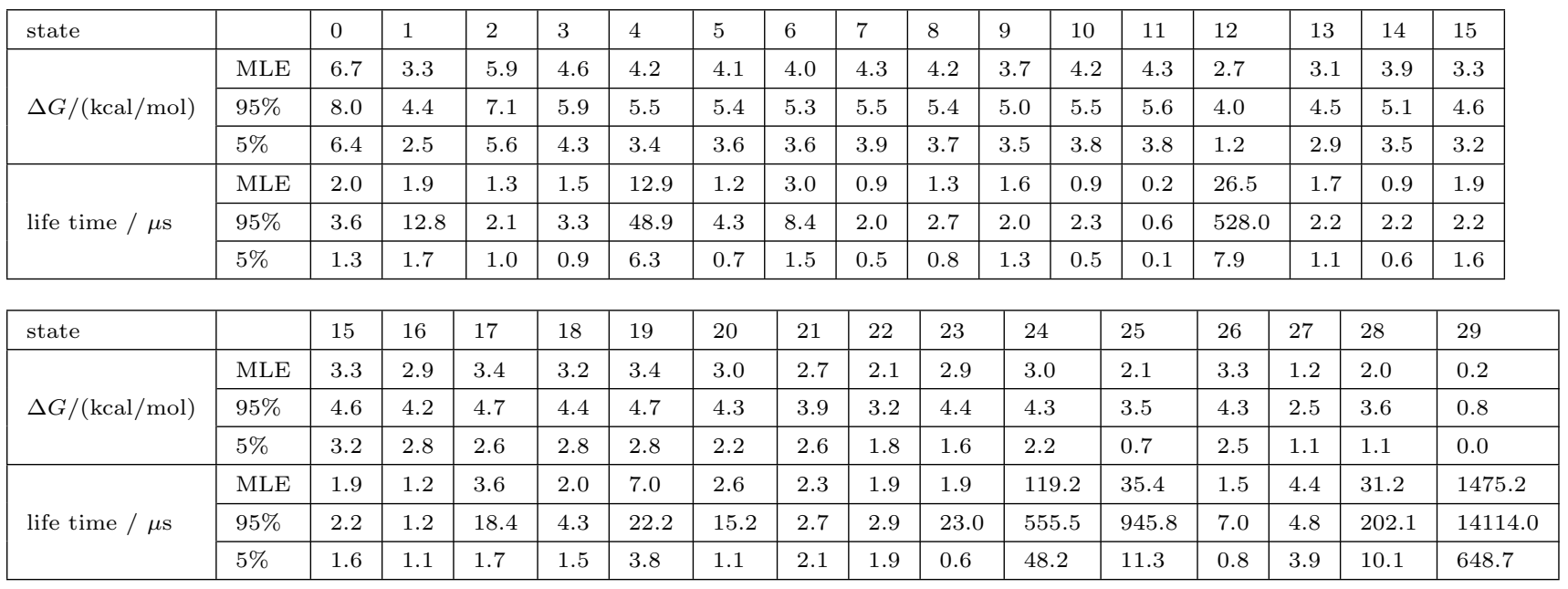

Suppl. Table 6: Free energies and life times of the PCCA states. For each state, the maximum likelihood estimate (MLE) of the free energy and the life time as well as their $5 \%$ and $95 \%$ credible intervals are reported. 


\begin{tabular}{|c|c|c|}
\hline macrostate $s$ & microstates needed to cover $90 \%$ of $\pi_{s}^{\prime}$ & total number of microstates \\
\hline 0 & 1 & 3 \\
\hline 1 & 819 & 2014 \\
\hline 2 & 50 & 99 \\
\hline 3 & 3 & 8 \\
\hline 4 & 3 & 5 \\
\hline 5 & 8 & 18 \\
\hline 6 & 1 & 3 \\
\hline 7 & 3 & 5 \\
\hline 8 & 1 & 2 \\
\hline 9 & 73 & 110 \\
\hline 10 & 2 & 6 \\
\hline 11 & 3 & 18 \\
\hline 12 & 2 & 7 \\
\hline 13 & 76 & 135 \\
\hline 14 & 5 & 10 \\
\hline 15 & 303 & 815 \\
\hline 16 & 354 & 695 \\
\hline 17 & 1 & 5 \\
\hline 18 & 14 & 37 \\
\hline 19 & 5 & 12 \\
\hline 20 & 3 & 9 \\
\hline 21 & 132 & 302 \\
\hline 22 & 554 & 2037 \\
\hline 23 & 3 & 4 \\
\hline 24 & 1 & 6 \\
\hline 25 & 1 & 18 \\
\hline 26 & 2 & 29 \\
\hline 27 & 1507 & 3522 \\
\hline 28 & 4 & 12 \\
\hline 29 & 4 & 25 \\
\hline
\end{tabular}

Suppl. Table 7: Compactness of macrostates measured by the minimal number of microstates that are needed to cover $90 \%$ of the probability mass of each microstate. 


\begin{tabular}{|c|c|c|c|c|c|}
\hline state & DFG & $\alpha \mathrm{C}[8]$ & $\alpha \mathrm{C}[3,12]$ & activity [6] & R-spine RMSD to $3 \mathrm{LCK} ; 1.8 \AA$ \\
\hline 0 & in & out & out & inactive & inactive \\
\hline 1 & out & out & out & inactive & inactive \\
\hline 2 & out & dilated & out-like & inactive & inactive \\
\hline 3 & out & out & out & inactive & inactive \\
\hline 4 & out & dilated & out & inactive & inactive \\
\hline 5 & - & in & in & active & inactive \\
\hline 6 & - & out & eut & inactive & inactive \\
\hline 7 & out & out & out & inactive & inactive \\
\hline 8 & out & out & out & inactive & inactive \\
\hline 9 & out & out & out & inactive & inactive \\
\hline 10 & - & in & in & inactive & active \\
\hline 11 & neither & out & out & inactive & inactive \\
\hline 12 & out & out & out & inactive & inactive \\
\hline 13 & out & out & out & inactive & inactive \\
\hline 14 & - & out & eut & inactive & inactive \\
\hline 15 & out & out & out & inactive & inactive \\
\hline 16 & in & in & in & active & active \\
\hline 17 & - & eut & eut & inactive & inactive \\
\hline 18 & out & out & out & inactive & inactive \\
\hline 19 & in & out & out & inactive & inactive \\
\hline 20 & in & out & out & inactive & inactive \\
\hline 21 & out & out & out & inactive & inactive \\
\hline 22 & out & out & out & inactive & inactive \\
\hline 23 & in & out & out & inactive & inactive \\
\hline 24 & out & dilated & out & inactive & inactive \\
\hline 25 & neither & out & out & inactive & inactive \\
\hline 26 & neither & out & out & inactive & inactive \\
\hline 27 & in & out & out & inactive & inactive \\
\hline 28 & in & out & out & inactive & inactive \\
\hline 29 & - & eut & eut & inactive & inactive \\
\hline
\end{tabular}

Suppl. Table 8: Structure-based estimates of activity for the 30 metastable states. In the third and fourth column, the criteria from ref. [8] $\left(d\left(\mathrm{C}_{\alpha}\right.\right.$-ASP-381, $\mathrm{C}_{\alpha}$-GLU-288 $\left.)<9 \AA\right)$ and Refs. [3, 12] $\left(d\left(\mathrm{C}_{\alpha}\right.\right.$-PHE-382, $\mathrm{C}_{\alpha}$-GLU-288 $\left.)<7.2 \AA\right)$ are used. In column five, the multivariate classifier from ref. [6] was applied to the snapshots form the MD simulation. In column 6, we use the average RMSD of R-spine atoms from the active reference conformation of crystal structure 3LCK. We call states with average R-spine RMSD $<1.8 \AA$ active. States with mis-folded N-lobe are crossed out.

\begin{tabular}{|c|c|c|c|c|c|}
\hline & & & \multicolumn{3}{|c|}{ Crystal structures at $x$ RMSD $^{*}$ around the state } \\
\hline$s$ & DFG & P-loop & $0 \AA$ to $2.5 \AA$ & $2.5 \AA$ to $3.0 \AA$ & $3.0 \AA$ to $3.5 \AA$ \\
\hline$\overline{0}$ & 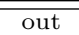 & ext. & & 4FG8, 5ZWJ, 2VTT, 1SNX, 3FZP + & 3RCD, 2R3N, 3BPR, 2VTN, 3CEK + \\
\hline 1 & - & - & & & \\
\hline 2 & out & kinked & & 3PYY, 3CS9 & 3PG3, 6GTT, 3OXZ, 3IK3, 2E2B + \\
\hline 3 & out & ext. & & & $6 \mathrm{FDZ}, 4 \mathrm{FG} 8$ \\
\hline 4 & out & ext. & & & 5U8L, 5HG5, 5UGA, 5HG7, 5HG9 + \\
\hline 5 & out & kinked & & & 5TO8, 3H3C, 3ET7, 3FZR, 5D9K + \\
\hline 6 & - & - & & & $3 \mathrm{R} 7 \mathrm{Q}, 2 \mathrm{CHX}, 5 \mathrm{ZV} 2,5 \mathrm{O} 49,3 \mathrm{~T} 8 \mathrm{M}+$ \\
\hline 7 & in & disordered & & & 2BDJ, 4D1S, 3KXZ, 5VD0, 2Y4P + \\
\hline 8 & - & - & & & \\
\hline 9 & in & ext. & & 3V5J, 3GGF, 4PP9, 1SM2, 3V5L + & 5JKG, 1SNX, 3QGY, 5XFF, 4KIO + \\
\hline 10 & out & ext. & & $2 \mathrm{HZO}$ & 2P2I, 5JFS, 3LFF, 5JFX, 5AR7 + \\
\hline 11 & in & ext. & & 5ZWJ, 5D41, 2VTT, 1SNX, 4IM0 + & 2W05, 1SNU, 3LZB, 1SM2, 3QGW + \\
\hline 12 & out & ext. & & & 3LFF, 6DKG, 3BE2, 3FZS, 4ZJJ + \\
\hline 13 & in & ext. & 1BUH, 1OIT, 5YU9, 4KD1, 3LZB + & 2W9F, 4HCU, 5VFI, 5IEY, 5P9G + & $3 \mathrm{V04}, 4 \mathrm{ZP} 5,5 \mathrm{DBX}, 5 \mathrm{O} 2 \mathrm{~B}, 5 \mathrm{JT} 2+$ \\
\hline 14 & out & kinked & 3 CS9 & 3PYY, 3OXZ, 2E2B, 3IK3, 5HU9 + & 5JFS, 6DKW, 5JFV, 3GVU, 5JFW + \\
\hline 15 & in & ext. & & 3LZB, 5D41 & $1 \mathrm{SNX}, 3 \mathrm{LZB}, 5 \mathrm{ZWJ}, 2 \mathrm{VTQ}, 1 \mathrm{SNU}+$ \\
\hline 16 & out & ext. & $3 \mathrm{H} 3 \mathrm{C}, 5 \mathrm{TO} 8$ & 3FZR, 3ET7, 1P14, 2VTN, 3FZP + & 2VTQ, 4FZA, 4O0R, 5TOB, 5D9K + \\
\hline 17 & inter & disordered & & & \\
\hline 18 & - & - & & & $4 \mathrm{ZP} 5,4 \mathrm{U} 41,4 \mathrm{U} 42,4 \mathrm{AAA}, 4 \mathrm{OBO}+$ \\
\hline 19 & - & - & & $3 \mathrm{U} 6 \mathrm{H}$ & \\
\hline
\end{tabular}

Suppl. Table 9: Same as main text table 2 but using a decomposition into 20 metastable PCCA states. 


\begin{tabular}{|c|c|c|c|c|c|}
\hline & \multicolumn{3}{|c|}{ Crystal structures at $x$ RMSD* ${ }^{*}$ around the state } \\
\hline$s$ & DFG & P-loop & $0 \AA$ to $2.5 \AA$ & \begin{tabular}{|c|}
$2.5 \AA$ Ato $3.0 \AA$ \\
\end{tabular} & $3.0 \AA$ to $3.5 \AA$ \\
\hline 0 & in & kinked & & $4 \mathrm{U} 42,4 \mathrm{ZK} 5,4 \mathrm{OBP}, 4 \mathrm{OBQ}, 2 \mathrm{CHZ}+$ & 4GB9, 4RWJ, 2CHX, 5EDS, 2QOH + \\
\hline 1 & in & kinked & & 3U6H, 4U42, 4ZP5, 5DI1, 5J95 + & $4 \mathrm{U} 41,4 \mathrm{OBO}, 4 \mathrm{ZSA}, 3 \mathrm{HUB}, 4 \mathrm{AOJ}+$ \\
\hline 2 & out & kinked & 3PYY, 3CS9, 2E2B, 3OXZ, 3IK3 & 3GVU, 2E2B, 3MS9, 3CS9, 3DZQ + & 3PG3, 1FPU, 2HIW, 6GTT, 6DKW + \\
\hline 3 & out/inter & coil & & $4 \mathrm{AF} 3$ & 6GTT, 3IK3, 3OXZ, 2E2B, 3PG3 + \\
\hline 4 & out & kinked & & & $4 \mathrm{OBO}, 4 \mathrm{U} 41$ \\
\hline 5 & out & kinked & & 3CS9, 3PYY & 6GTT, 3PG3, 4U42, 3OXZ, 4AF3 + \\
\hline 6 & out & kinked & & 3PYY, 3CS9 & 6GTT, 3PG3, 3OXZ, 3IK3, 4AF3 + \\
\hline 7 & out & kinked & $3 \mathrm{PYY}$ & 2E2B, 3CS9, 3OXZ, 3IK3, 4AF3 & 3PG3, 3GVU, 2E2B, 3DZQ, 5HU9 + \\
\hline 8 & inter & coil & & & 4AF3, 3DJ6 \\
\hline 9 & out & kinked & $3 \mathrm{PYY}, 2 \mathrm{E} 2 \mathrm{~B}$ & 3OXZ, 3CS9, 3IK3, 4AF3 & 3PG3, 3GVU, 2E2B, 3DZQ, 5HU9 + \\
\hline 10 & out & ext. & & 4FG8, 5ZWJ, 2VTT, 1SNX, 3FZP + & 2R3N, 3BPR, 3CEK, 4FK3, 3RCD + \\
\hline 11 & - & - & & & \\
\hline 12 & out & ext. & & & $4 \mathrm{ZJJ}$ \\
\hline 13 & - & - & & & \\
\hline 14 & out & ext. & & 3FZS, 2OG8, 3LFF, 6DKG, 3BE2 & 2OG8, 4BKJ, 6FNG, 6DKB, 2BAK + \\
\hline 15 & out & ext. & $6 \mathrm{FNG}$ & 2OG8, 3FZS, 6FNJ, 3LFF, 3BE2 + & 4BKJ, 6DKB, 4F7N, 4ZJJ, 4F6S + \\
\hline 16 & out & disordered & & & \\
\hline 17 & - & \begin{tabular}{|l|}
- \\
\end{tabular} & $5 \mathrm{NKA}$ & 5NK0, 3QGY, 3R7Q, 2REI, 5NKI + & 3DBS, 4GK2, 5IA3, 2A5U, 3L13 + \\
\hline 18 & in & coil & & & 3H3C, 3FZP, 1P14, 5TO8 \\
\hline 19 & out & ext. & & & 6FDZ, 4FG8 \\
\hline 20 & out & ext. & $2 \mathrm{HZ} 0,2 \mathrm{P} 2 \mathrm{I}$ & 3LFF, 2QU5, 5AR7, 5JFS, 4X7H + & 4F7L, 6DKB, 3BE2, 6DKG, 4EQU + \\
\hline 21 & - & - & & & \\
\hline 22 & in & disordered & & & \\
\hline 23 & out & \begin{tabular}{|c|} 
ext. \\
\end{tabular} & & $5 \mathrm{TO} 8,3 \mathrm{H} 3 \mathrm{C}$ & 3ET7, 3FZR, 2XNP, 5D9K \\
\hline 24 & in & disordered & & 4ZTL, 3PXK, 4GK2, 4D1S, 2DQ7 + & 4RX8, 4RX7, 3SRV, 4XG9, 1QPJ + \\
\hline 25 & out & \begin{tabular}{|c|} 
ext. \\
\end{tabular} & & 4ZJV, 4I20, 5HG5, 5UGA, 5U8L + & 2RF9, 4USD, 4I21, 4HNI, 4ZTL + \\
\hline 26 & - & - & & & \\
\hline 27 & in & ext. & & 3V5J, 4PP9, 3GGF, 3V5L, 1SM2 + & 5JKG, 4KIO, 5XFF, 1SNX, 3QGY + \\
\hline 28 & in & ext. & 1SNX, 1SNU, 3QGW, 3QGY, 4LUE + & 3VS4, 3VRY, 3VS3, 3VS5, 4LUD + & 1FMK, 4IM3, 5FP5, 3DKC, 1AQ1 + \\
\hline 29 & in & ext. & & 5ZWJ, 5D41, 2VTT, 1SNX, 4IM0 + & 1SM2, 3QGW, 3LZB, 3EZV, 3C4C + \\
\hline 30 & out & ext. & & & $1 \mathrm{BYG}$ \\
\hline 31 & in & ext. & & 3BRB, 1SNU, 3QGW, 1SNX, 1SM2 + & 4FK3, 4IM2, 4G34, 4IM0, 6B16 + \\
\hline 32 & out & ext. & & & 3LFF, 6DKG, 4ZJJ, 3BE2, 5JFX + \\
\hline 33 & in & ext. & 1BUH, 1OIT, 4KD1, 5YU9, 4EK8 + & 4RG0, 5H2U, 5GTY, 5D7V, 3VS4 + & 4ARK, 3ZLW, 3OS3, 2B52, 5BX0 + \\
\hline 34 & out/inter & kinked & 3CS9 & 3PYY, 3CS9, 3OXZ, 2E2B, 3IK3 + & 5JFS, 6DKW, 3GVU, 5JFV, 5JFW + \\
\hline 35 & in & ext. & & 5D41, 3LZB, 2BTR, 1SNX, 2VTQ + & 4K2R, 4FG8, 3OG7, 2R3L, 1GIH + \\
\hline 36 & out & ext. & $3 \mathrm{H} 3 \mathrm{C}, 5 \mathrm{TO} 8$ & 3FZR, 3ET7, 1P14, 2VTN, 3FZP & 5D9K, 2VTQ, 4O0R, 4FZA, 5TOB + \\
\hline 37 & inter & kinked & & & \\
\hline 38 & - & - & & $3 \mathrm{U} 6 \mathrm{H}$ & $4 \mathrm{ZP} 5,4 \mathrm{U} 41,4 \mathrm{U} 42,4 \mathrm{AAA}, 4 \mathrm{OBO}+$ \\
\hline 39 & - & - & & & \\
\hline
\end{tabular}

Suppl. Table 10: Same as main text table 2 but using a decomposition into 40 metastable PCCA states. 


\begin{tabular}{|c|c|c|c|}
\hline metastable state & P loop & DFG cluster & DFG subcluster \\
\hline 0 & kinked & in ... & $\ldots$ BBA- $\ldots$ noise \\
\hline 1 & kinked & in ... & noise \\
\hline 2 & kinked & out & $\ldots$ BBA- $\ldots$ noise \\
\hline 3 & coil & out inter & - BBA- noise \\
\hline 4 & kinked & out & noise \\
\hline 5 & kinked & out & $\ldots$ BBA- ... noise \\
\hline 6 & kinked & out & 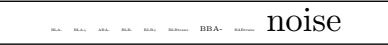 \\
\hline 7 & kinked & in out & $\ldots \ldots$ BBA- $\ldots$ noise \\
\hline 8 & coil & in inter & . n noise \\
\hline 9 & kinked & out & BBA- $\ldots$ noise \\
\hline 10 & extended & in out & noise \\
\hline 11 & - & in ... & BLA- $\ldots \ldots \ldots$ \\
\hline 12 & extended & in out _ & $\ldots$ BBA- $\ldots$ noise \\
\hline 13 & - & in $\ldots$ & вLA $\mathrm{BL}+\ldots \ldots$ \\
\hline 14 & extended & out & BBA- $\ldots$ noise \\
\hline 15 & extended & out & $\ldots$ BBA- \\
\hline 16 & disordered & in Out inter & $\ldots$ BBA- вавtrans noise \\
\hline 17 & - & in $\ldots$ & BLA- $\ldots$ \\
\hline 18 & coil & in out niner. & . \\
\hline 19 & extended & out & $\ldots$ BBA- $\ldots$ noise \\
\hline 20 & extended & in out _ & ................ nisA- noise \\
\hline 21 & - & in out inter & BABtrans noise \\
\hline 22 & disordered & in & BLA- BLA+ ABA- ……...... noise \\
\hline 23 & extended & in out & 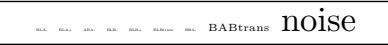 \\
\hline 24 & disordered & in out - & BLA- \\
\hline 25 & extended & out & $\ldots$ BBA- $\ldots$ noise \\
\hline 26 & - & in $\ldots$ & ........ BLB $+\ldots$ \\
\hline 27 & extended & in ... & $\ldots$ BLB $+\ldots \ldots$ \\
\hline 28 & extended & in $\ldots$ & ........ \\
\hline 29 & extended & in $\ldots$ & $\mathrm{BLA}+\ldots \mathrm{BLB}+\ldots \ldots$ \\
\hline 30 & extended & Out inter & $\ldots \ldots$ BBA- $\ldots$ noise \\
\hline 31 & extended & in $\ldots$ & 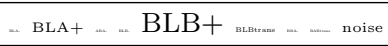 \\
\hline 32 & extended & in out & $\ldots \ldots \ldots \ldots$ BBA- $\ldots$ noise \\
\hline 33 & extended & in $\ldots$ & BLB $+\ldots$ \\
\hline 34 & kinked & out inter & $\ldots \ldots$ BBA- $\ldots$ noise \\
\hline 35 & extended & in $\ldots$ & $\ldots$ вLA+ _... BLB $+\ldots \ldots$ noise \\
\hline 36 & extended & out & . noise \\
\hline 37 & kinked & inter & noise \\
\hline 38 & - & in $\ldots$ & $\mathrm{BLA}+\ldots \mathrm{BLB}+\ldots \ldots$ \\
\hline 39 & - & in ... & 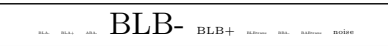 \\
\hline
\end{tabular}

Suppl. Table 11: Same as suppl. Tab. 2 but using a decomposition into 40 metastable states. Misfolded states that were excluded from the analysis are marked with a dash in the P loop column. 


\begin{tabular}{|c|c|c|c|}
\hline metastable state & P loop & DFG cluster & DFG subcluster \\
\hline 0 & extended & in out - & noise \\
\hline 1 & - & in $\ldots$ & BLA- ... \\
\hline 2 & kinked & in Out inter & $\ldots \ldots \ldots$ BBA- $\ldots$ noise \\
\hline 3 & extended & out & $\ldots$ BBA- $\ldots$ noise \\
\hline 4 & extended & Out inter & 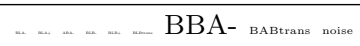 \\
\hline 5 & kinked & in Out inter $_{\text {in }}$ & вABtrans noise \\
\hline 6 & - & in $\ldots$ & BLA- BLA+ $\ldots \ldots$ \\
\hline 7 & disordered & in _...ter & BLA- ${ }_{\mathrm{B} L++} \mathrm{ABA}-\ldots \ldots$ noise \\
\hline 8 & - & in $\ldots$ & $\ldots \mathrm{BLB}+\ldots \ldots$ \\
\hline 9 & extended & in $\ldots$ & BLB $+\ldots \ldots$ \\
\hline 10 & extended & in out - & 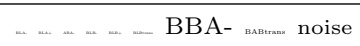 \\
\hline 11 & extended & in $\ldots$ & $\mathrm{BLA}+\ldots \mathrm{BLB}+\ldots \ldots$ \\
\hline 12 & extended & in Out inter & $\ldots$ BBA- $\ldots$ noise \\
\hline 13 & extended & in $\ldots$ & 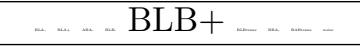 \\
\hline 14 & kinked & out inter & $\ldots \ldots$ BBA- $\ldots$ noise \\
\hline 15 & extended & in out & 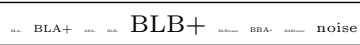 \\
\hline 16 & extended & out & 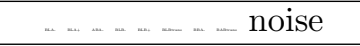 \\
\hline 17 & disordered & inter & noise \\
\hline 18 & - & in $\ldots$ & $\mathrm{BLA}+\ldots \mathrm{BLB}+\ldots \ldots$ \\
\hline 19 & - & in $\ldots$ & BLB- вцв+ $\ldots \ldots \ldots$ \\
\hline
\end{tabular}

Suppl. Table 12: Same as suppl. Tab. 2 but using a decomposition into 20 metastable states. Misfolded states that were excluded from the analysis are marked with a dash in the $\mathrm{P}$ loop column.

\begin{tabular}{|c|c|c|c|c|c|c|c|}
\hline ad hoc state & DFG & P-loop & $\alpha \mathrm{C}$ & A-loop & origin & (A) PCCA state & (B) PCCA state \\
\hline \hline 1 & in & kinked & in & ext. & PDB: 2F4J & 16 & 16 \\
\hline 2 & in & ext. & in & ext. & PDB: 2GQG & 16 & 16 \\
\hline 3 & out & kinked & in & beta & PDB: 2IEP & 1 & 1 \\
\hline 4 & out & kinked & in & ext. & PDB: 1 M52 & 15 & 15 \\
\hline 5 & out & ext. & in & beta & by homology & 22 & 22 \\
\hline 6 & out & ext. & out & ext. & PDB: 3 UE4 & 15 & 15 \\
\hline 7 & in & kinked & out & alpha & Frankenstein & 29 & 27 \\
\hline 8 & in & kinked & out & ext & by homology & 27 & 16 \\
\hline 9 & in & ext. & out & alpha & PDB: 2 GIT & 26 & 27 \\
\hline 10 & in & ext. & out & ext & by homology & 29 & 27 \\
\hline 11 & out & kinked & out & alpha & by homology & 27 & 1 \\
\hline 12 & out & kinked & out & beta & Frankenstein & 22 & 15 \\
\hline 14 & out & kinked & out & ext & PDB: 2 G2H & 22 & 21 \\
\hline 15 & out & ext. & out & alpha & Frankenstein & 21 & 1 \\
\hline 16 & out & ext. & out & beta & by homology & 2 & 5 \\
\hline
\end{tabular}

Suppl. Table 13: In this table we relate the ad hoc macrostates 1 to 16 from ref. [7] to the 30 metastable states of our current article. In ref. [7], the representatives of the ad hoc states ("cluster centers") were manually selected to reflect the various conformational combinations of the flexible structural elements (DFG, P-loop, $\alpha \mathrm{C}, \mathrm{A}-\mathrm{loop}$ ) by using Abl crystal structures if the given combination was crystallized or by taking recourse to homology and "Frankenstein" models where no corresponding crystal exists. The 7th column (A) shows the PCCA state that overlaps the most with a given ad hoc state (overlap between states were computed with a $\pi$-weighted scalar product of the indicator functions). The last column (B) shows the PCCA state into which the ad hoc cluster center falls. Matching entries in columns A and B are highlighted in red. Comparing columns A with B shows that the majority of ad hoc states are inconsistent, i.e. the cluster center is not located in the metastable states that maximally overlaps with the ad hoc state. That's likely because of the very simple definition of ad hoc states that does not capture the true features of Abl's free energy landscape. We observe the general trend that cluster centers which are crystal structures produce more consistent definitions than homology models or "Frankenstein" models. 


\section{Suppl. References}

[1] M. P. Harrigan, M. M. Sultan, C. X. Hernández, B. E. Husic, P. Eastman, C. R. S., K. A. Beauchamp, R. T. McGibbon, and V. S. Pande. MSMBuilder: Statistical models for biomolecular dynamics. Biophys. J., 112(1):10$15,2017$.

[2] Bettina G. Keller, Andrei Kobitski, Andres Jäschke, G. Ulrich Nienhaus, and Frank Noé. Complex RNA folding kinetics revealed by single-molecule FRET and hidden Markov models. J. Am. Chem. Soc., 136(12):4534-4543, 2014.

[3] Albert J. Kooistra, Georgi K. Kanev, Oscar P.J. van Linden, Rob Leurs, Iwan J.P. de Esch, and Chris de Graaf. KLIFS: a structural kinase-ligand interaction database. Nucleic Acids Res., 44(D1):D365-D371, 102015.

[4] James A. Maier, Carmenza Martinez, Koushik Kasavajhala, Lauren Wickstrom, Kevin E. Hauser, and Carlos Simmerling. ff14SB: Improving the accuracy of protein side chain and backbone parameters from ff99SB. $J$. Chem. Theory Comput., 11(8):3696-3713, 2015.

[5] Robert T. McGibbon, Kyle A. Beauchamp, Matthew P. Harrigan, Christoph Klein, Jason M. Swails, Carlos X. Hernández, Christian R. Schwantes, Lee-Ping Wang, Thomas J. Lane, and Vijay S. Pande. MDTraj: A modern open library for the analysis of molecular dynamics trajectories. Biophys. J., 109(8):1528-1532, 2015.

[6] Daniel Ian McSkimming, Khaled Rasheed, and Natarajan Kannan. Classifying kinase conformations using a machine learning approach. BMC Bioinformatics, 18(1):86, 022017.

[7] Yilin Meng, Cen Gao, David K. Clawson, Shane Atwell, Marijane Russell, Michal Vieth, and Benoît Roux. Predicting the conformational variability of Abl tyrosine kinase using molecular dynamics simulations and Markov state models. J. Chem. Theory Comput., 14(5):2721-2732, 2018.

[8] Henrik Möbitz. The ABC of protein kinase conformations. BBA-Proteins Proteom., 1854(10, Part B):1555-1566, 2015.

[9] Vivek Modi and Roland L. Dunbrack. Defining a new nomenclature for the structures of active and inactive kinases. Proc. Natl. Acad. Sci. USA, 116(14):6818-6827, 2019.

[10] Martin K. Scherer, Benjamin Trendelkamp-Schroer, Fabian Paul, Guillermo Pérez-Hernández, Moritz Hoffmann, Nuria Plattner, Christoph Wehmeyer, Jan-Hendrik Prinz, and Frank Noé. PyEMMA 2: A software package for estimation, validation, and analysis of Markov models. J. Chem. Theory Comput., 11:5525-5542, 2015.

[11] David W Scott. Multivariate density estimation: theory, practice, and visualization. John Wiley \& Sons, Hoboken, New Jersey, U.S.A., 2015.

[12] Oscar P. J. van Linden, Albert J. Kooistra, Rob Leurs, Iwan J. P. de Esch, and Chris de Graaf. KLIFS: A knowledge-based structural database to navigate kinase-ligand interaction space. J. Med. Chem., 57(2):249-277, 2014. 